\title{
Dual protection in an integrated community-based program: A case study of Tanzania Family Health/Ministry of Health Project in Mbeya
}

Grace Mbekem

Jane Chege

Population Council

Follow this and additional works at: https://knowledgecommons.popcouncil.org/departments_sbsr-rh

Part of the Community Health and Preventive Medicine Commons, Demography, Population, and Ecology Commons, International Public Health Commons, and the Maternal and Child Health Commons How does access to this work benefit you? Let us know!

\section{Recommended Citation}

Mbekem, Grace and Jane Chege. 1998. "Dual protection in an integrated community-based program: A case study of Tanzania Family Health/Ministry of Health Project in Mbeya." Kenya: TFHP and Population Council. 


\section{DUAL PROTECTION IN AN INTEGRATED COMMUNITY-BASED PROGRAM: A CASE STUDY OF TANZANIA FAMILY HEALTH/MINISTRY OF HEALTH PROJECT IN MBEYA}

Paper Presented at the Workshop on Improving Quality of Care: Guidelines and Standards for Reproductive Health. Harare, Zimbabwe 8 - 12 June 1998

Grace Mbekem (MCommH) $\mathrm{MCH}$ Coordinator

TFHP

P. O Box 2561

Mbeya

Telephone: (255) 65 - 4089

Fax:

(255) $65-4172$

Email: frank-fhp@maf.org
Jane Chege (Ph.D.)

Population Council/

GRHeP Consultancy

P. O Box 28266

Nairobi

(254) 2710651

(254) 2710651

grhep@africaonline.co.ke 


\subsection{BACKGROUND}

\subsection{Introduction}

One of the most pressing challenges for health programs in most Sub-Saharan African countries is how to effectively address the increasing prevalence of HIV/AIDS. Recent evidence suggests that controlling sexually transmitted diseases (STDs) through undertaking preventive measures, early diagnosis, and treatment significantly slows the spread of HIV/AIDS. In regard to STI/HIV, Maternal and Child Health $(\mathrm{MCH})$ and Family Planning (FP) clients are described as "low risk" groups. However, in a number of Sub-Saharan African countries, the reported levels of STDs are significantly high to justify use of limited resources to target this group for STD services. MCH/FP Programs have recently begun to look into ways of getting more involved in prevention of STDs/HIV among MCH and FP clients. This is being addressed through designing integrated programs and providing integrated $\mathrm{MCH} / \mathrm{FP}$ and STD/HIV services. This move towards integration is gaining momentum.

However, integration of STD/HIV/AIDS activities into MCH/FP services presents immense challenges for reproductive health programs in the region. The issue of the best cost-effective strategy to provide quality integrated $\mathrm{MCH} / \mathrm{FP} / \mathrm{STD} / \mathrm{HIV}$ services in the context of scarce financial, laboratory, and technical resources is still unsettled. Among the uncertainties facing integration is the technical challenge on how to alter the existing $\mathrm{MCH} / \mathrm{FP}$ services so as to simultaneously meet contraceptive and disease control goals. One of the most effective approaches identified to meet this dual challenge is to rely more heavily on barrier methods. Currently, the male condom is the most effective barrier method. In this context it can be used in conjunction with other FP methods or alone for the dual purpose of protecting against pregnancy and STD/HIV infection.

Integrated reproductive health services are provided in various Sub-Saharan countries in varying degrees and service mix. In some programs, only $\mathrm{MCH} / \mathrm{FP}$ activities are integrated with STI/HIV services. Other programs integrate a wider range of reproductive health services. Although not all programs in the Sub-Saharan region provide integrated reproductive health services, service integration is taking place both at the static health facilities and at the community-based level. Some countries have developed national FP guidelines and standards to guide service providers. For some countries, these guidelines and standards have implicit or explicit instructions on how to provide integrated reproductive health services. For example, the revised ${ }^{1}$ Kenya Ministry of Health (MOH) FP policy guidelines and standards are now "Reproductive Health/Family Planning Policy Guidelines and Standards For Service Providers". These provide guidelines and standards not only on FP services but also on Maternal Health/Safe Motherhood, Post Abortion Care, and integration of STI/HIVAIDS control into the MCH/FP program.

\footnotetext{
${ }^{1}$ These were first published in 1991 and revised in June 1997.
} 


\subsection{The Tanzania Family Health Project}

Since October 1994, the Tanzania Family Health Project (TFHP) has been providing financial and technical support to the Ministry of Health to improve reproductive health services in five districts in Mbeya Region. The project is jointly funded by the Government of Tanzania and the UK Department For International Development (DFID).

- The project aims to improve the access and increase utilization of high quality integrated family health services with particular emphasis on services for family planning (FP) and the control of sexually transmitted infections (STIs).

- In order to achieve this goal, the Project is involved in improving the quality of services provided at the health facilities. In addition, the Project is increasing community demand and access for services through use of community members as reproductive health service providers.

- Mbeya region was selected as the priority project region because of its low contraceptive prevalence rate (CPR), high infant and maternal mortality, population growth and incidence of STIs. In HIV/AIDS prevalence Mbeya Region is second to Dar es Salaam.

\section{Project Activities}

The Project implements activities at both the health facility and community level.

Health Facility Level: The following activities are implemented:

- Staff clinical skills training in FP and STI service delivery

- Service quality training

- Improve supervision through the training of regional and District Health Management Teams (DHMTs) in supervisory skills

- Improve infrastructure through renovating the buildings with community participation

- Supply of clinical and non-clinical equipment

- Community Level: The community-based activities use varying groups of community actors who provide complimentary health services and act as a link between the health facilities and the community. The main activities implemented at the community level are:

- Train Traditional Birth attendants (TBAs), community- based Distribution (CBD) agents, Village Primary Health Care Committee (PHCC) members and Ward Training Teams (WTTs) to provide some elements of reproductive health services. TBAs focus mainly on safe motherhood. WTTs and CBD agents focus mainly on IEC on FP and STI. In addition to IEC, CBD agents distribute non-clinical FP methods and referral services for clinical methods and STI diagnosis and treatment. The WTTs also 
provide IEC on malaria prevention and community participation in management, financing, and use of health services.

- The Project is in advanced stages of designing a reproductive health program to specifically target the youth.

\section{The Role of WTTS}

The WTT is composed of three volunteer members. These are selected from different government sectors (that have a community focus ${ }^{2}$ ) and the community (village health workers or influential community members). The members of their community select them using criteria provided by the project.

Their role is to:

- Create community awareness of Project activities

- Strengthen the community sense of ownership and their participation in management of health services

- Improve the knowledge of disease and other related problems prevalent in the community

- Sensitize and mobilize the community to utilize FP and STI services offered at both the health facility and community-based service delivery points.

- Strengthen the relationship between community health actors (CBDs, TBAs, VHWs), health facility staff and the community.

- Train community health actors on use of participatory rural appraisal (PRA) methodology.

To achieve their objectives WTTs use the following strategies:

- Have formal and informal discussions with community individuals and groups and through public meetings (barazas).

- Identify influential individuals referred to as community own resource persons (CORPS) and train them to pass FP and STI information and education to their contacts

- Revive VPHCC that are not effective

- Strengthen the involvement of VPHCC in management of health services and promotion of health information

The main reason for the WTTs initiative is to allow for sustainability of the health service management and provision of community-based health services after the Project life.

\footnotetext{
${ }^{2}$ For example, the Ministries of Education, Agriculture, Water, Social services, etc.
} 


\section{The Role of CBD Agents}

The CBD initiative started as a pilot project in ten Wards of one project district (Mbozi). Twenty CBD agents were selected by the community and received training on reproductive health services delivery in late 1996. After the evaluation of the pilot program in October 1997, the program is now expanded to other Wards in Mbozi district and other Project districts. The CBD agents undertake the following activities:

- IEC on FP, STI/HIV prevention and Safe Motherhood and Child Survival

- Distribute pills, condoms and spermicides

- Refer clients for clinical FP services, screen for pill use, STI diagnosis and treatment and safe motherhood.

\subsection{Community-Based Integration of FP and STI Services and Promotion of Dual Protection}

To implement its services, the TFHP/MOH uses the integrated service delivery approach through training service providers on this approach. The Project uses the national FP policy guidelines and standards, training curricular and protocol manuals. To assess CBD agents promotion of dual protection in their provision of integrated reproductive health services, we used the following data collection methods:

- Review of CBD agents' client registers and work plans

- Questionnaire interviews with CBD agents to assess their attitudes, knowledge and practices related to dual protection

- Focus group discussions with groups of CBD agents

- Individual in-depth interviews with the CBD supervisors and program staff

- Review of the national standards and guidelines, CBD curriculum, CBD protocol manual, IEC materials used by the agents, CBD supervisors training curriculum and supervision manual 


\subsection{Tanzania FP National Policy Guidelines and Standards}

The revised ${ }^{3}$ Tanzania MOH National Policy Guidelines and Standards For Family Planning Service Delivery and Training focus mainly on FP services. However, these guidelines include STD/HIV/AIDS prevention as part of the package of services to provide to FP clients both at the health facility and at the community-based service delivery points. The guidelines highlight the importance of providing information on dual protection.

Among other requirements, the guidelines and standards stipulate that:

- All males and females of reproductive age, irrespective of their parity and marital status, shall have the right to FP information, education, and services. The guidelines underline adolescents, women over 35 years of age, women who gave birth less than two years ago and men as the sub-population groups to receive priority.

- These services are to be provided through health facilities, "with special emphasis on" MCH and FP clinics, and through community-based and commercial marketing program outlets.

- Supervision of family planning services ....."will be integrated in the existing supervision system for $\mathrm{MCH}$ services and other related PHC services". P. 4.

- While counseling and screening clients for use of FP methods, health facility providers and all community-based and social marketing FP providers will educate individuals and couples on how to protect themselves from STDs and HIV/AIDS and receive training on methods that prevent STDs and HIV/AIDS transmission.

- At the health facilities, clients will be screened for use of FP methods through relevant reproductive history that includes among other things the client's sexual history. In screening for use of contraceptive method by examination and laboratory tests, in addition to examination/tests essential for various contraceptives use, the guidelines recommends examination/test not necessary for contraceptive method but necessary for reproductive health screening. Screening and serological testing for STDs is recommended for all hormonal method and intrauterine device use.

- At the community Based Distribution (CBD) or social marketing services, the clients' health and social history shall be obtained through use of an approved simple checklist.

- Clients selecting barrier methods of contraception shall be informed that "correct use is necessary for the effectiveness of the method and that "“The condom helps to prevent STDs, HIV and AIDS".

\footnotetext{
${ }^{3}$ These were first published in 1992 and revised in 1994.
} 
- Providers should provide information on dual purpose of condoms (for protection against pregnancy and STD/HIV infection) to clients who condom is their method of choice.

\subsection{Dissemination of the FP National Policy Guidelines at the CBD Level}

The national standards and guidelines are disseminated through the training of CBD agents and their supervisors and through supervision. The guidelines and standards are incorporated in the national CBD:

- Training curriculum

- Protocol manual

- Supervisors training curriculum

- Supervision manual and checklist

The CBD agents were trained using the earlier integrated reproductive health curriculum. This curriculum does not underline promotion of dual method use as a way of promoting dual protection amongst clients who are using contraceptive methods other than condoms. However, the revised national curriculum for training CBD agents (April 1997) clearly underlines the importance of dual method use.

The CBD protocol manual, used by agents as a reference material, provides detailed information of the services to provide to the community. Of interest, the protocol stipulates that CBD agents provide information about all FP methods. Apart from condoms, agents are required to provide information that other contraceptive methods do not protect against STI/HIV infection. For the condom, clients should be informed of their dual purpose, that is, they prevent unwanted pregnancy and protect against STI/HIV infection.

CBD supervisors receive training on supervision of all aspects of CBD work. During their supervision, they are required to use a supervisory checklist that contains 20 sections each dealing with a specific area. The checklist allows for comprehensive supervision of the following aspects:

- Work plans

- Record keeping and reporting

- Group and individual education

- Counseling of new and continuing clients

- Referral

- Contraceptive requisition and storage 


\subsection{Implementation of Guidelines and Standards in CBD Service Provision}

Due to this wide dissemination of the national FP policy guidelines and standards, CBD agents clearly understand and implement services according to the guidelines and standards they have received. Their understanding was demonstrated in their responses during the focus group discussions (FGD) and individual interviews. The CBD activities were assessed to examine the extent to which they follow the established guidelines and standards particularly in relation to STI/HIV prevention.

\section{Right of Access to FP Services}

- A review of CBD work plans for the month of January to March 1998, show CBD agents plan to provide IEC to various categories of people. The work plans targeted

1. Mixed male and female of child bearing age groups

2. Youth groups

3. Women groups

4. Church groups

5. Special groups such as traditional dancing groups, drama groups

6. Home-visits

- A review of the CBD client registers indicated that the agents provide contraceptive services to both male and female clients of varying ages and marital status.

- Male clients mainly use condoms

- The age of the clients ranged from 15 to 46 years

- Most male clients are single

- Most female clients are married

\section{Priority to Adolescents and Men}

- During the focus group discussions with CBD agents, they reported that they target both adolescents and men. During their home-visits, they target not only the women but also their partners. They offer FP and STI prevention information initially to the couple. If the male partner is absent, they book an appointment to visit when the partner is at home. They provide information to the youth when they meet them in groups and those interested in contraceptives receive confidential counseling and services.

- Out of the fifteen agents who were interviewed individually:

- 10 had unmarried adolescent clients

- 14 were aware of the policy guidelines to provide FP and STI/HIV health services to adolescents 
- In response to the questions as to what they would do if an unmarried girl or an unmarried boy aged between 15 to 24 years requested FP and STI services from them,

- 10 said they would provide FP services to the girl

- 10 said they would provide condoms for STI prevention to the girl

- 13 said they would provide FP services to the boy

- 14 said they would provide condoms to the boy for STI prevention.

In view of the social-cultural barriers towards provision of services to unmarried youth, it is remarkable that the highest percentage of these agents expressed a positive attitude towards providing services to the youth. A similar assessment of CBD agents' attitudes in Kenya showed a strong gender bias in provision of contraceptive services and generally, a negative attitude towards providing services to the youth. While 81 percent of the agents said they would provide services to an unmarried adolescent boy, only 26 percent said they would provide the same to a girl of similar characteristics (Chege \& Askew, 1997). This striking difference in attitude between Kenyan and TFHP programs' CBD agents is probably due to the differences in policy guidelines, training and in the age of the CBD agents recruited. While Tanzania has a very clear policy on targeting the youth, the Kenyan policy guidelines do not clearly focus on adolescents ${ }^{4}$. The majority of the TFHP CBD agents are aged between 22 and 28 years. It is likely that their comparatively young age contributes to their positive attitude to providing services to the youth.

\section{Condom Clients to Receive Information on Dual Purpose}

- During the FGDs, the agents reported that they provide information on dual purpose of condoms not only to the condom clients but to all clients. Agents are required to provide information about all the FP methods to all their new clients.

\section{All FP Clients to Receive Education on STI/HIV Prevention}

- CBD agents have received training in common signs and symptoms, modes of transmission and ways to prevent the spread of STIs and HIV/AIDS

- The CBD protocol manual contains relevant information required for STI/HIV counseling

- During the FGDs CBD agents reported that they provide information and counseling services on STI/HIV to their clients. However, a review of agents' records revealed few referrals for STI services. Further, agents do not remember to provide information on STI/HIV information to clients as much as they remember other essential information they should provide to new clients. Agents were asked to state all the information they provide to new FP clients. Compared to other FP information, where over a half of the agents mentioned spontaneously,

\footnotetext{
${ }^{4}$ The revised policy guidelines (1997) underline the importance of providing information to the youth but are silent on provision of contraceptives.
} 
only three out of the 15 agents interviewed spontaneously mention STI/HIV prevention. However, after probing, all the agents said that they provide information on STI/HIV prevention.

\section{All Hormonal Method and IUCD Users to be Screened or Tested for STI}

- All CBD agents have a checklist for screening new pill users. This checklist contains eleven question one of which relates to vaginal discharge.

- The checklist stipulates that any client who responds positively to any one of these questions should receive condoms and referred to the health facility

\section{Supervision of CBD Activities}

- CBD supervision is attached to the health facility within the CBD catchment area. The immediate CBD supervisor is usually the $\mathrm{MCH}$ Aide or Rural medical Aid whose activities are in turn supervised by the district $\mathrm{MCH}$ coordinator (DMCH). The monthly reports on CBD activities are sent to the DMCH coordinator.

- However, during the quarterly supervision of health facilities by the District Health management Team (DHMT), there is no room for the DMCH coordinator to supervise the CBD agents. Her supervision is limited to the level of the health facility with the immediate CBD supervisor. The supervisory checklists used by the DHMT do not have any section for supervising CBD activities.

\section{Promotion of Condom and Dual Method Use}

The CBD and WTT IEC activities do promote condom use at the community level. This is done through providing information on the use of condom as one of the major ways of preventing STI/HIV transmission. As mentioned earlier, the CBD agents also distribute condoms.

- Review of client registers of the 15 agents who were interviewed indicated that 34 percent of all their clients were using condoms.

- Comparatively, the TFHP/MOH CBD program is one of the highly effective programs in condom distribution in the East African region. The average annual distribution of condoms per CBD agent per year is among the highest. Table 1.1 presents a comparison of the average condom client visits per CBD amongst seven CBD programs in Kenya and Tanzania. Both the Nairobi City Council Family Planning Project (NCCFP) in Kenya and the Seventh Day Adventist (SDA) program in Tanzania rely on full time paid agents providing services mainly in the urban areas. Among programs relying on volunteer agents, the TFHP/MOH agents have the highest average condom client visits and CYP. 
Table 1.1 Annual Average Condom client Visits and CYP per CBD By Program

\begin{tabular}{|l|l|l|}
\hline Program & Condom client visits & Condom CYP \\
\hline NCCFP $^{5}(1994)$ & 1999.7 & 164 \\
\hline TFHP $(1997)$ & 246 & 29.6 \\
\hline NCCS $^{6}(1994)$ & 133.4 & 27.1 \\
\hline MYWO $^{7}(1994)$ & 71.8 & 13.6 \\
\hline FLPS $^{8}(1994)$ & 69.8 & 16.8 \\
\hline UMATI $^{9}(1995 / 6)$ & 52.6 & 5.8 \\
\hline SDA $^{10}(1995 / 6)$ & 17.6 & 8.1 \\
\hline
\end{tabular}

- A comparison of trends in condom client visits in clinics in Mbozi district that have CBD sites in their catchment areas and those that do not have, indicates that CBD agents contribute significantly to the level of condom use. As indicated in Figure 1.1, the reported number of client visits in ten clinics that have CBD sites is significantly higher with the introduction of the CBD approach in 1997.

\section{Fig. 1 Condom Client Visits in SDPs with \& SDPs Without CBD Agents (1995-1997)}

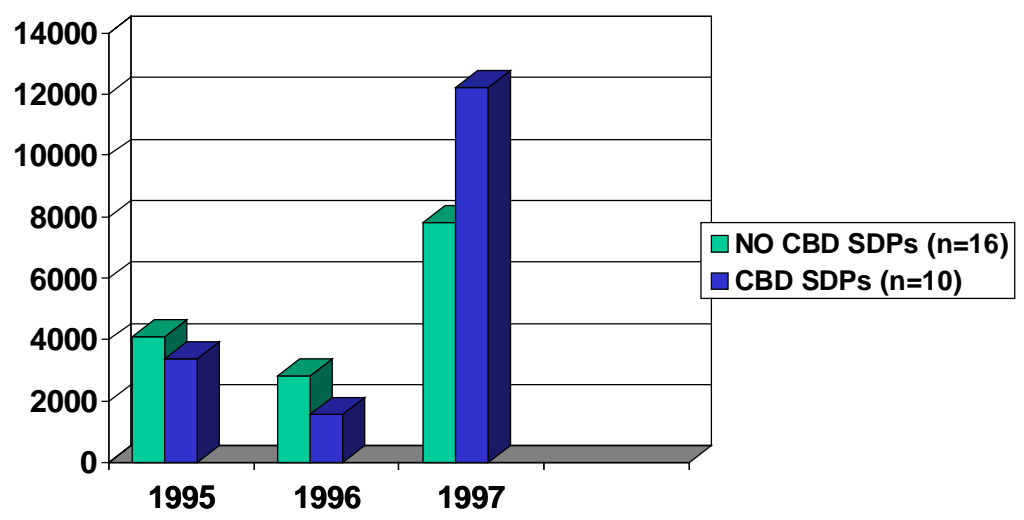

\footnotetext{
${ }^{5}$ Nairobi City Council Family Planning Project (Kenya)

${ }^{6}$ Nairobi City Council Urban Slum Project (Kenya)

${ }^{7}$ Maendeleo Ya Wanawake Organization (Kenya)

${ }^{8}$ Family life Promotion Serices (Kenya)

${ }^{9}$ Tanzania Family Planning Association

${ }^{10}$ Seventh Day Adventist Project (Tanzania)
} 
- Although the national FP policy guidelines and standards do not lay emphasis on promotion of dual method use, some CBD clients use dual methods. This is mainly because those clients who assess themselves as being at risk of STI/HIV infection do use condoms even when they are using another contraceptive method.

- CBD agents have not received any training on assessing clients who are at risk of STI/HIV infection so as to encourage them to use condoms. In response to the question on what they would do to help an FP client who appears to be at risk of STI/HIV infection, only two agents said they would ask her to continue with her current method but also supply her with condoms.

\section{Conclusion}

The findings of this CBD assessment indicate that given the proper training and guidance, CBD agents would promote dual protection method use. It is also suggested that the addition of community- based reproductive health providers is partly responsible for the significant increase in the number of condoms distributed in Tanzania and the rise of condom use in Mbeya region. The latest Tanzania Demographic and Health Survey (1996) indicate that Mbeya has the highest percentage of men reporting condom use in the country. Nationally, 7 percent of the males reported that they use condom while in Mbeya the percentage was 25. It is therefore important to explore modalities of increasing the capacity of CBD agents in promoting dual protection and dual method use.

In addition, the research findings indicate the importance of clear well disseminated policy guidelines in guiding provision of services. As the case of providing services to adolescents indicate, with proper training, there is a strong possibility of changing provider attitudes. 


\section{References}

Chege, Jane and Ian Askew, 1997. An assessment of Community-Based Family Planning Programmes in Kenya. The Population Council, Africa Operations Research and Technical Project II Report.

Chege, Jane, N. Rutenberg, B. Janowitz and A. Thompson. 1998. Factors Affecting the Outputs and Costs of Community-Based Distribution of Family Planning Services in Tanzania. The Population Council, Africa Operations research and Technical Assistance Project II Report.

Family Health Project, First Annual Report 1994/1995.

Family Health Project, Annual Report 1995/1996

Family Health Project, Annual Review: 1996

Kuyoh, M. 1997. "Use of Male Condom For FP and STI/HIV Prevention in Kenya: A Review of Published and Unpublished Literature". FHI

Ministry of Health Tanzania, September 1997. Curriculum For Training CBD Supervisors.

Ministry of Health, National Family Planning Programme Tanzania. April 1997. National Curriculum For Training Community-Based Distributors.

Ministry of Health, National Family Planning Programme Tanzania, 1997. Mwongozo Wa Msimamizi.

Ministry of Health, National Family Planning Programme Tanzania, 1997. Mwongozo Wa Mhudumu.

Ministry of Health, Tanzania. 1994. National Family Planning Policy Guidelines.

Welsh, M. P. Feldblum and S. Chan. 1997. Sexually Transmitted Disease Risk Assessment Used Among Low-Risk Populations in East /Central Africa: A Review in East African Medical Journal Vol. 74 no 12, December 1997. 